\title{
La Acreditación Institucional de la Calidad en los Institutos Superiores Técnicos y Tecnológicos del Ecuador desde la Perspectiva de un Instituto Acreditado
}

\author{
Víctor G. Gómez, Manuel R. Tolozano y Noemí B. Delgado \\ Instituto Superior Tecnológico Bolivariano de Tecnología, Víctor Manuel Rendón 236 y Pedro Carbo, \\ Guayaquil, Ecuador (e-mail: vgomez@bolivariano.edu.ec; rtolozano@bolivariano.edu.ec; \\ ndelgado@bolivariano.edu.ec)
}

Recibido Abr. 7, 2017; Aceptado Jun. 21, 2017; Versión final Ago. 28, 2017, Publicado Dic. 2017

\begin{abstract}
Resumen
Se presenta, desde dos perspectivas, la global y la particular, las experiencias, debilidades y fortalezas del proceso de evaluación institucional los centros de Educación Superior Técnicas y Tecnológicas en Ecuador. Se aborda críticamente las implicaciones y aprendizajes que ha dejado este proceso con la intencionalidad de iniciar el camino para la discusión académica sobre el tema. Estas reflexiones se agrupan en varios momentos: la cultura de la calidad en el Sistema de Educación Superior ecuatoriano; la realidad de la evaluación, los mecanismos y modelos desde la perspectiva del Instituto Bolivariano; la preparación de los Institutos Superiores Técnicos y Tecnológicos para la Acreditación Institucional; la racionalidad del modelo aplicado y la instrumentación del proceso de evaluación y de mejora continua posterior a la evaluación. Se concluye que el proceso de evaluación puede contribuir al mejoramiento de la eficiencia y eficacia académicas de las instituciones acreditadas o en proceso de acreditación.
\end{abstract}

Palabras clave: acreditación institucional; calidad en educación superior; institutos tecnológicos superiores

\section{The Institutional Accreditation of Quality in Technical and Technological Institutes of Higher Education in Ecuador from the Perspective of an Accredited Institute}

Institutional accreditation of quality in Technical and Technological Institutes of Higher Education is analyzed from two perspectives: global and particular. The study critically addresses the implications and learning that have been developed as a result of this certification with the intention of starting the path for academic discussion on this subject. These reflections are grouped into several steps: the culture of quality in higher education system in Ecuador, the reality of the evaluation mechanisms and models during its implementation from the perspective of the Bolivariano Technological Institute, the qualification of the Technical and Technological Institutes for institutional accreditation, the rationality of the applied model and the instrumentation used in the evaluation process, and the continuous improvement after the evaluation. It is concluded that the evaluation process may improve the academic efficiency and effectiveness in accredited higher education institutes.

Keywords: institutional accreditation; quality in higher education; higher technological institutes 


\section{INTRODUCCIÓN}

Con toda intencionalidad, en las líneas que siguen se tratará de abrir un sendero hacia la reflexión en torno a la dificultad que entraña la emisión de juicios de valor desde la complejidad de las comparaciones objetivas y subjetivas entre sujetos o entre este y el entramado social que lo alberga como parte del sistema educativo ecuatoriano que, como todo proceso institucionalizado, se debate a pesar de los avances, los esfuerzos y las acciones, entre persistentes brechas en temas de acceso, de calidad, de resultados de la educación en general y de la educación superior en particular.

Según la Ley Orgánica de Educación Superior (LOES), el Consejo de Evaluación, Acreditación y Aseguramiento de la Calidad de Educación Superior (CEAACES) ejerce por ley “(...) la rectoría de la política pública para el aseguramiento de la calidad de la educación superior del Ecuador a través de procesos de evaluación, acreditación y categorización en las IES" (Asamblea Nacional del Ecuador, 2010). El artículo 94 de la LOES deja claro que la evaluación de la calidad de una institución, carrera o programa académico es un proceso mediante el cual se realiza la "recopilación sistemática de datos cuantitativos y cualitativos que permitan emitir un juicio o diagnóstico, analizando sus componentes, funciones, procesos, a fin de que sus resultados sirvan para reformar y mejorar el programa de estudios, carrera o institución" (Asamblea Nacional del Ecuador, 2010) y resalta que es "un proceso permanente y supone un seguimiento continuo".

Hopkins (1994) define la mejora de una institución como un enfoque para el cambio educativo que lleva a planos superiores los resultados de los estudiantes y fortalece la capacidad para gestionar el cambio y recomienda el abordaje del entorno de éstas como unidad de cambio, mejora, innovación y desarrollo. Los conceptos que ofrece la literatura especializada sobre la evaluación de la calidad de las instituciones de educación superior (IES) adolecen, unos más que otros, de indefiniciones desde el punto de vista epistemológico, metodológico y práctico. Según Becket y Brookes (2008), los procesos de aseguramiento de la calidad se han convertido en instrumentos preponderantes dentro de las políticas de educación superior en el mundo destacando que existe falta de consenso respecto a la mejor manera de gestionarla.

En este sentido el propio CEAACES reconoce que "La evaluación de las instituciones de educación superior (IES) está incluida entre los problemas que se definen como vagamente estructurados debido, entre otras razones, a la conectividad entre los criterios que se deben tener en cuenta (básicamente por escasez de recursos), la ambigüedad de los conceptos (el propio concepto de calidad de la educación superior), heterogeneidad de los objetos de evaluación (instituciones universitarias y tecnológicas, públicas y privadas, etc.), naturaleza diferente de la información a utilizar (cuantitativa y cualitativa) y ausencia de métrica y unidad común en los indicadores" (CEAACES, 2016).

A criterio de los autores de este trabajo, para entender el entramado de direcciones que toma el proceso de acreditación en el mundo y la diversidad de posiciones que se asumen de un sistema de educación superior a otro se necesita definir las bases generales sobre la cual se asienta la calidad de la educación superior para poder adentrarse en la conceptualización de otro término no menos importante que el anterior: la evaluación de la calidad de la educación superior. Entonces, se hace necesario dejar claro que para los autores de este trabajo, el término "calidad de la educación superior" es inherente y está estrechamente vinculado al contexto y los escenarios en que subiste la IES y a la "misión" como estandarte de su filosofía institucional y no un estándar absoluto generalmente influenciado por las voces externas que asesoran la implementación de los modelos en un sistema de educación superior u otro. Ello coincide con las opiniones vertidas por Tyler y Bernasconi (1999) cuando aseveran que "el patrón de medida de la evaluación (...) debe ser aquello que la institución es razonablemente capaz de lograr dada su misión específica y su realidad presente" e insisten en que "(...) Si una universidad (...) recluta a estudiantes académicamente desaventajados, que presentan déficits importantes en sus aptitudes intelectuales básicas, el criterio de calidad para esa institución debe ser el valor agregado, no el valor absoluto del producto final". Este constituye la debilidad primera del modelo de evaluación aplicado a los ISTT en Ecuador, desde su propia filosofía interna de ser un modelo de benchmarking hasta las reglas únicas e iguales para todas las instituciones.

Estos razonamientos sustenta la tesis planteada por Redon (2009) en cuanto a que el concepto de calidad no es un concepto inequívoco sino que está delimitado e influenciado por ideologías, intereses y valores lo que es perfectamente evidenciable en muchos de los sistemas de educación superior actuales donde la evaluación institucional no solo es vista como un proceso de mejora continua sino también como un proceso de control interno y externo que limita por momentos la autonomía de las IES. Hoyos (2016), por su parte, dice que la calidad en las instituciones educativas es más "un concepto relativo a la sociedad a la que pertenecen las instituciones de educación superior, que (...) algo definible por sí mismo". 
Natarajan (2000) hace recomendaciones sobre evaluación de la calidad académica relativa declarando que debe ser multidimensional, que debe basarse en aspectos medibles y que debe considerar los logros de los profesores y los estudiantes, entre aspectos. Así mismo, estos procesos transformadores de la calidad institucional deben considerar: la heterogeneidad de los evaluados, las debilidades y sesgos del proceso mismo y de los evaluadores, la flexibilidad para facilitar la contraposición de puntos de vistas, el acceso transparente a la información, la identificación de las debilidades, la definición de las mejoras a implementar, la claridad en la determinación de las líneas base y las garantías de que las metas se proyecten dentro de un proceso caracterizado por la búsqueda de la excelencia, de la eficiencia y de la comparación constante con los mejores y que apunte, prospectivamente, hacia el perfeccionamiento permanente. Un ejemplo de modelo poco complejo desde el punto de vista de su aplicación pero congruente y efectivo para valorar de una manera simple la eficiencia de las funciones de docencia e investigación desde una perspectiva financiera lo constituye el propuesto por Ramírez y Alfaro (2013) para el caso de las universidades pertenecientes al Consejo de Rectores de las Universidades Chilenas.

Al existir una significativa diversidad de definiciones para el término "calidad de la educación superior" la literatura especializada reporta diversos modelos de evaluación y autoevaluación institucional. Stufflebeam (1994) en su artículo "Recomendaciones para mejorar las evaluaciones en las escuelas públicas de Estados Unidos" publicado en la revista Studies in Educational Evaluation expresa que no siempre se aplican verdaderos modelos de evaluación de la calidad educativa en tanto algunos marcan una distancia significativa en relación a la naturaleza misma del acto de emisión de juicios de valor sobre un desempeño determinado y, en ese sentido, los clasifica en modelos pseudoevaluativos, cuasievaluativos y evaluativos.

Por su parte, House (2014) realiza un ordenamiento interesante que involucra a los modelos de evaluación de la calidad educativa. Este autor cita ocho categorías de modelos que a su vez reduce a tres grandes grupos a partir de los elementos comunes que los caracterizan. En este sentido, incluye en un primer grupo a aquellos modelos que a pesar de que parten de un exhaustivo proceso de recopilación de información y evidencias significativas transmiten los resultados de modo selectivo o parcial. En el segundo grupo se propone una serie de modelos que solo tienden a resolver un determinado problema, identificado con anterioridad sin pronunciarse con respecto al comportamiento o la calidad global de la organización. En el tercer grupo analiza la inclusión de los modelos de evaluación de la calidad que apuntan a una visión integral de los sistemas educativos y particulariza, desprejuiciadamente, en los elementos institucionales con el propósito de proyectar su mejora teniendo en cuenta la filosofía del benchmarking y, con ello, la posibilidad de conocer quien tiene desempeños estandarizados, quien y mediante qué acciones superó el estándar de un criterio determinado y, por último, como debe ser proyectada la mejora con estándares de desempeño que hagan competitivamente mejor a la institución educativa en un entorno donde otros demostraron poder alcanzar altos niveles de resultados.

Tyler y Bernasconi (1999) aseguran que no puede haber un patrón o estándar de calidad aplicable a todas las instituciones y que el sistema de evaluación debe ser capaz de manejar diversos criterios de excelencia. Ello complejiza la labor de los órganos de acreditación y evaluación de la calidad y de los modelos mismos que se aplican pero, al mismo tiempo, los profesionaliza. Los mencionados autores consideran que "la ley debe abstenerse de legislar calidad, y ello por dos razones: primero, porque es perfectamente inútil, pura letra muerta, dado que la calidad no se impone por decreto" (Tyler y Bernasconi, 1999).

En ese sentido, la calidad de la educación superior está determinada por un conjunto de procesos transformadores delimitados por la complejidad del entorno y de los escenarios mismos en los que se desempeñan las IES. Entonces, la evaluación de la calidad de la educación superior se estructura a partir de la emisión de juicios de valor comparados que demuestren los cambios cualitativos y/o cuantitativos desde diferentes ángulos de una problemática autoidentificada internamente. Los autores de este trabajo consideran como puntales para la emisión de juicios de valor comparados: la evolución de los procesos de enseñanza y aprendizaje; la pertinencia en el cumplimiento del encargo social de las IES; las metodologías, modelos, procedimientos, modalidades e innovaciones generadas o reproducidas y, por último, los niveles de efectividad institucional, de eficiencia y eficacia en la gestión de los procesos sustantivos, de los procesos de apoyo y de los procesos especiales que tengan lugar en la IES y la integración entre ellos.

\section{RACIONALIDAD DEL MODELO Y DEL PROCESO DE EVALUACIÓN}

La literatura especializada da cuenta de estudios recientes realizados acerca de los efectos y la efectividad de los procesos de evaluación de la calidad sobre los sistemas de educación superior de diferentes países. Por ejemplo Harvey y Williams (2010) concluyen que los sistemas de aseguramiento de la calidad no mejoran realmente la educación superior. Estos autores insisten en el hecho de que se evidencian muy pocas mejoras en las IES donde la acreditación y evaluación de la calidad se vinculan a ejercicios de rendiciones de cuentas. 
Para el caso de los impactos de estos procesos en Europa, Loukkola (2010) indica que las estructuras de apoyo a la calidad en las IES en la Unión Europea no son permanentes y su vida inicia y culmina con el propio proceso evaluativo por lo que en muchos casos no están institucionalizadas y agrega que la información resultante de los procesos de evaluación no siempre es accesible pues es considerada confidencial al interior de las IES y desde los órganos evaluadores externos hacia las IES. Algo parecido sucede en el sistema de evaluación y acreditación de la calidad en el Ecuador. Lemaitre y Zenteño (2012) realizaron un estudio similar en países iberoamericanos destacando que los resultados de la evaluación desarrollada en el marco de los sistemas de aseguramiento de la calidad pasan a convertirse en insumos relevantes para la planificación de la mejora institucional. Países como Cuba y Estados Unidos con procedimientos similares de acreditación complementan la autoevaluación institucional con una revisión externa. Las IES realizan una autoevaluación para la mejora de la calidad y luego, mediante la constatación in situ por expertos externos se emite el resultado final. En el Reino Unido, por su parte, los procesos de acreditación y evaluación de la calidad tienen como objetivo comprobar que la información que publican las instituciones de educación superior sobre la calidad y los estándares que han alcanzado es real, exacta y transparente. Lo expuesto reafirma la tesis de que el impacto de los procesos de evaluación con fines de acreditación sobre las funciones sustantivas y la gestión institucional varía según el tipo de política aplicada y el nivel de institucionalización de los procesos.

Con regularidad los diferentes modelos que se implementan, sin variaciones significativas de un país a otro o de una región a otra, se basan en las consideraciones convencionales de la teoría de los sistemas en relación a las entradas, las transferencias y las salidas o la matriz insumo-proceso-producto lo que posibilita agrupar ordenadamente los momentos, funciones y componentes del proceso de gestión institucional permitiendo visualizar la relación existente entre los mismos. Comúnmente, en los modelos se definen "dimensiones o criterios" de calidad. En el caso de dimensiones resaltan: relevancia, efectividad, disponibilidad de recursos, eficiencia, eficacia y procesos. Para criterios, como en el caso del modelo genérico aplicado por el CEAACES, se incluyen con frecuencia los criterios: Pertinencia, Currículo, Calidad de la docencia, Infraestructura y Ambiente institucional.

En una IES la mayor parte de la actividad que se desarrolla está referida directamente al proceso de formación del profesional, sin embargo, el modelo aplicado por el CEAACES para las instituciones universitarias y no universitarias privilegia, de manera especial, los criterios de Calidad de la Docencia e Infraestructura con pesos específicos de 0,3834 y 0,2979, respectivamente. Profundizando en el análisis de estos criterios puede verse que, a pesar de que constituyen el $40 \%$ del total de los criterios del modelo, el peso específico asignado a ambos representa el 68,13\%. Cabe preguntarse: ¿a qué elementos hacen referencia? ¿Se considera en ellos la función de docencia o formación del profesional concebida no sólo como la relación profesor-alumno dentro del proceso de enseñanza y aprendizaje e incluye la investigación, la extensión y gestión? ¿Se tiene en cuenta en el criterio infraestructura todos los elementos que intervienen en la asignación de recursos y valora la calidad del gasto, la eficiencia y la sostenibilidad en lo invertido?

En el modelo aplicado por el CEAACES a los ISTT, el criterio Calidad de la Docencia parte de la consideración de que el docente es el "recurso más importante que una institución incorpora en el proceso de formación, porque su desempeño impacta de manera preponderante en la calidad de todos los resultados de la actividad institucional" (CEAACES, 2016) y lo coloca en el centro del criterio desde las dimensiones siguientes: suficiencia, estructura de su carga laboral, selección, evaluación, idoneidad de su formación y experiencia, remuneración, formación, nivel de actualización profesional y producción de conocimientos. En este sentido: ¿es el docente el elemento clave de la calidad educativa? Efectivamente, tener profesores competentes, motivados y bien entrenados, capaces de desarrollar pedagogías y currículos pertinentes es un buen punto de partida en temas de "calidad" pero está más que demostrado, que la "calidad de la educación" no está relacionada únicamente con la calidad de los docentes. Por ejemplo un currículo fuerte, diseñado con maestría desde lo pedagógico, que posibilite los aprendizajes y los favorezca desde la lógica de las ciencias tiene impacto en la calidad de la formación profesional y, por lo tanto, en la calidad del producto final que se entrega como parte del encargo social, mediante el graduado.

Por su parte, con el criterio Infraestructura se pretende en el modelo caracterizar "la base material de que dispone el ISTT para el desarrollo del proceso de enseñanza-aprendizaje y para garantizar apropiadas condiciones de vida en la institución a los docentes, trabajadores y estudiantes" (CEAACES, 2016) entre las que se pueden mencionar la dotación y el estado de las bibliotecas, aulas, oficinas para docentes, el acceso a internet, la disponibilidad de ambiente virtual para la interrelación entre directivos, docentes y estudiantes, el estado de los laboratorios de informática y otros laboratorios o talleres específicos de las diferentes carreras, las medidas de seguridad para evitar accidentes y enfermedades laborales, la accesibilidad para personas con discapacidad física, el estado de los servicios higiénicos y las condiciones generales para el descanso, socialización y esparcimiento hacia el interior de la institución. La disponibilidad de recursos en la que enfatiza el modelo del CEAACES trata de identificar los medios con los que cuenta el ISTT para cumplir 
sus compromisos en el corto y mediano plazo pero olvida algo tan importante como el patrimonio institucional, aun cuando la LOES en su disposición transitoria décima primera, establece que "El patrimonio (...) deberá cubrir, al menos, los pasivos tangibles e intangibles, considerando como prioritario la cobertura de los derechos de las estudiantes a completar su carrera cumpliendo con los requisitos académicos regulares" (Asamblea Nacional del Ecuador, 2010).

Aparecen entonces algunas interrogantes acerca de la omisión del tema patrimonial: ¿tendría relación con el hecho de que varios ISTT utilizan infraestructura física de terceros? ¿Serían tantos en esta situación que considerarlo en el modelo de evaluación pondría en riesgo los resultados finales? Los autores prefieren dejar solamente formuladas estas interrogantes, en definitiva, valorando las aseveraciones de Rendon (2009): la calidad es un concepto que está delimitado e influenciado por ideologías, intereses y valores.

Por otro lado, ni el modelo de evaluación de los ISTT ni el aplicado a las universidades para la categorización incluyen, en relación a la disponibilidad de recursos con que cuenta la institución, un análisis elemental de cómo son utilizados los mismos en función de los resultados. Los indicadores no profundizan en la eficiencia y la calidad del gasto, aspecto tratado abundantemente por Ramírez y Alfaro (2013) y descrito arriba. Podría decirse que en el Criterio Infraestructura no midió la cantidad y calidad de los recursos invertidos para alcanzar los resultados sino que, a criterio de los autores, solamente se establecieron los patrones para el cumplimiento de los estándares mínimos de calidad lo que es incongruente con un modelo sustentado en la filosofía del benchmarking y con el crecimiento del gasto público en educación superior que casi se duplicó del 2009 al 2015 según fuentes del Ministerio de Finanzas y la SENESCYT reportadas por Villacis (2016) en su análisis del financiamiento de 30 universidades públicas.

Llama la atención que en abril del 2016 el Diario el Telégrafo perteneciente a la empresa pública Telégrafo EP resalta que "Una de las falencias que aún se mantienen en las universidades es el gasto que destinan para cubrir las gestiones administrativas. Este asciende a 57\%, cuando la norma establece que sea el 35\%" (Diario El Telégrafo, 2016). Sin embrago, lo expresado no es consistente con lo expuesto meses antes por el economista René Ramírez, expresidente del Consejo de Educación Superior (CES), en el marco de un acto efectuado el lunes 12 de octubre de 2015 en las instalaciones de la Universidad de las Artes en Guayaquil. En el portal de la propia Universidad de las Artes se publicó el artículo "La educación superior en el Ecuador a cinco años de la LOES" donde se presentó un balance del estado de la educación superior en el país y se "felicitó a las universidades públicas por disminuir el gasto administrativo que pasó del 54\% al $35 \%$ del presupuesto, pero las desafió a mejorar el gasto a la medida de las universidades privadas que, en materia administrativa, gastan únicamente el $25 \%$ del presupuesto, permitiendo que los recursos se destinen a la mejora del sistema de educación, a la investigación y la academia" (Universidad de las Artes, 2015). En todo caso ambos hacen referencia a la calidad del gasto, elemento que junto a la transparencia de la ejecución presupuestaria, el valor de los activos y la existencia o no de patrimonio institucional no tuvieron la representatividad que, por ley, le correspondería dentro del modelo de evaluación de los ISTT.

Tabla 1: Resultados generales de la evaluación por tipo de ISTT

\begin{tabular}{|l|c|c|c|c|}
\hline $\begin{array}{c}\text { Parámetros } \\
\text { descriptivos }\end{array}$ & $\begin{array}{c}\text { Particular } \\
\text { autofinanciado }\end{array}$ & Público & $\begin{array}{c}\text { Particular } \\
\text { cofinanciado }\end{array}$ & Total \\
\hline Número & 104 & 107 & 8 & 219 \\
\hline Media & 0,50 & 0,35 & 0,35 & 0,40 \\
\hline Mediana & 0,50 & 0,33 & 0,38 & 0,40 \\
\hline Desviación Estándar & 0,26 & 0,16 & 0,25 & 0,22 \\
\hline Valor Máximo & 0,97 & 0,79 & 0,73 & 0,83 \\
\hline Valor Mínimo & 0,00 & 0.00 & 0,00 & 0.00 \\
\hline
\end{tabular}

Si se analizan los resultados generales de la evaluación que ofrece el CEAACES en el Informe General de la Evaluación de los Institutos Superiores Técnicos y Tecnológicos se tiene que los ISTT particulares autofinanciados, presentan, de manera general, un mejor desempeño que los de régimen público y los particulares cofinanciados. En la evaluación institucional participaron 219 ISTT de los cuales 107 eran públicos, 104 particulares autofinanciados y 8 particulares en régimen de cofinanciamiento (reciben fondos públicos para su gestión) y como se observa en la tabla 1, la media del desempeño de los ISTT particulares autofinanciados supera en 15 puntos porcentuales a los ISTT públicos y particulares cofinanciados. EI informe general no explica a qué se debe esta asimetría y trata de minimizar el hecho expresando que "Los valores de desviación estándar indican un comportamiento sensiblemente más uniforme de los institutos 
públicos $(0,16)$ con relación a los particulares, tanto autofinanciados como cofinanciados $(0,26$ y 0,25 respectivamente), estos dos últimos con similar comportamiento" (CEAACES, 2016a).

Una lectura diferente de los datos del informe muestra que, desde el punto de vista estadístico, entre los institutos particulares cofinanciados y autofinanciados existen algunos con buen desempeño y otros con bajo lo que se explica por la desviación estándar de 0,26. Así mismo, para el caso de los ISTT públicos con una desviación estándar de 0,16 que los hace "sensiblemente más uniformes", la calidad global de su gestión es cuestionable en tanto están agrupados mayoritariamente en la zona de bajos desempeños (la media para este grupo es de 0,35). Todo ello evidencia que cualquier conclusión en el ámbito de la evaluación institucional, no sólo debe basarse en determinados supuestos de naturaleza estadística o conceptual-metodológica, sino que debe evitar sesgos en la formulación misma de las conclusiones.

Dicho informe muestra que en los criterios de Pertinencia y Currículo existe al menos un instituto que alcanza el valor referencial del criterio $(1,00)$. Por otro lado, el mismo criterio de Pertinencia junto al de Ambiente Institucional, alcanzan deficientes resultados reflejando 0,0 y 0,0850 , respectivamente, en el cuartil Q25 (CEAACES, 2016a). El criterio Currículo alcanza los mejores desempeños de manera general por parte de los ISTT. Sin embargo, en el informe el CEAACES se recomienda que los valores que se exponen "permiten a cada instituto contrastar sus resultados relativos en cada criterio con diferentes niveles de desempeño relativos, desde el cuartil 25 (Q25), hasta el valor máximo alcanzado en cada criterio, incluyendo el promedio de todas las instituciones evaluadas" lo que solo es una valoración más, exclusivamente cuantitativa, sobre la distancia que le separa del mejor puntuado en cada criterio pues no se socializaron nunca los resultados obtenidos por cada ISTT sino que se emitieron informes individuales confidenciales y, por lo tanto, se limita el trabajo en red de un instituto "condicionado" con otro de mejor desempeño. Esto vuelve a poner en dudas el enfoque de benchmarking del modelo aplicado.

De manera general, el informe final emitió un juicio de valor sobre un nivel de calidad que existía desde un período de dos años antes de la fecha de la evaluación. No todos trabajaron a favor de la calidad de la misma manera, sin embargo, unos y otros quedaron dentro del proceso, los primeros como "acreditados", los segundos "condicionados" o "fuertemente condicionados" lo que se asimila por los primeros como desmotivador y desestimulante. Por otra parte, la disminución de los estándares a niveles tan bajos para que un ISTT resultara "no acreditados" (por debajo del $20 \%$ de desempeño) según el Artículo 11 del Reglamento para la determinación de resultados del proceso de evaluación (CEAACES, 2016b), es muestra de la aplicación de reglas discrecionales que desestimula a los comprometidos con la educación de calidad.

\section{RESULTADOS Y DISCUSION}

El Instituto Superior Tecnológico Bolivariano de Tecnología logra un desempeño de un $97 \%$ y los valores en cada uno de sus indicadores se muestran en la Figura 1.

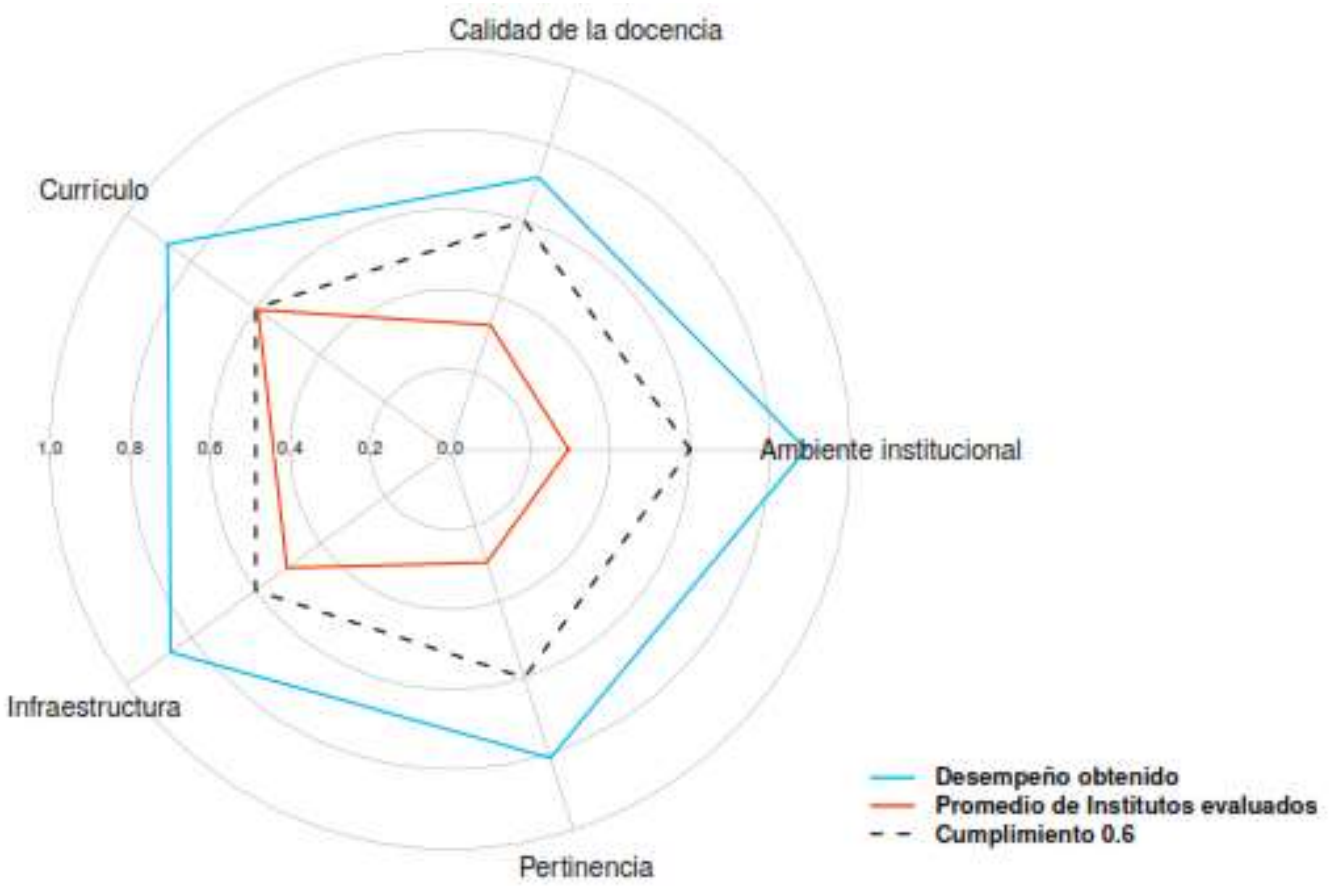

Fig. 1: Resultados generales de la evaluación de los ISTT por criterios del modelo 
En la Figura 1 se compara el desempeño alcanzado con la media de los resultados de los ISTT y el estándar de calidad definido por el modelo (CEAACES, 2016c). El desempeño del $97 \%$ alcanzado por el ITB lo ubica entre las instituciones acreditadas. En el instituto, los diferentes actores coinciden en que el proceso, independientemente de las debilidades que lo caracterizaron y que fueron mencionadas arriba, es útil ya que genera mecanismos de reflexión sobre el desempeño de los diferentes niveles de la estructura organizacional. Institucionalmente es aceptado que las estrategias implementadas para el mantenimiento y el perfeccionamiento del desempeño alcanzado en la evaluación, que coloca al ITB como líder entre los institutos superiores técnicos y tecnológicos del país, han generado cambios más sustantivos que reactivos en tanto provienen de fuerzas endógenas de la institución. En ese sentido, durante todo el proceso de preparación para la evaluación se generaron cambios fácticos y positivos en los indicadores relativos al claustro de profesores, currículo, eficiencia terminal, en varias dimensiones de la función investigación, en la gestión institucional, la infraestructura, los servicios de bienestar, las política de acción afirmativa, equidad, inclusión, bibliotecas, calidad del gasto, entre otros.

Los resultados que presenta el instituto y los del proceso de acreditación de ISTT, proponen un desafío hacia la única agencia de acreditación del Ecuador: demostrar la efectividad y el impacto que la acreditación tiene sobre los profesionales graduados, sobre los estudiantes en proceso de formación, el papel que la acreditación debe jugar en la selección de la IES y la carrera por parte de los bachilleres para acceder a la Educación Superior así como la forma que dichos resultados deben incidir en los procesos de homologación, equivalencia y transferencia de créditos o tiempos de dedicación (Morse, 2008) para la movilidad estudiantil y la continuidad de estudios. En resumen, se necesita continuar trabajando por demostrar la credibilidad social de la acreditación desde la perspectiva de la mejora de los procesos y los resultados educativos.

\section{CONCLUSIONES}

De lo anterior se deduce que persiste la necesidad sustantiva de: 1) insistir en que los procesos de evaluación de la calidad de la educación no deben responder a cambios en la gobernabilidad, la institucionalidad, ni tener sesgo político o ideológico; 2) definir procesos de autoevaluación y evaluación con reglas claras y estables, equitativas, no discrecionales y transparentes que consideren la realidad del entorno y de los ISTT de manera particular; 3) minimizar la subjetividad de los pares pues ello atenta contra la calidad del proceso al imponerse la lógica personal y la experiencia individual sobre lo objetivo y el modelo mismo; 4) visibilizar propuestas e instrumentos explícitos de apoyo y estímulo a las IES que finalizan con altos desempeños la evaluación institucional de manera que se estimule a los "acreditados" a lograr empeños superiores y a la mejora continua; 5) resolver los problemas de ineficiencia del sistema de educación superior ecuatoriano para gestionar los procesos en los ISTT públicos.

Las IES ecuatorianas no debían conformarse con la aplicación de mecanismo y modelos lineales intensivos, intrusivos y caros heredados de la lógica positivista sino que deben enrumbarse los proceso futuros bajo la premisa de que la calidad sólo puede lograrse donde existe compromiso, responsabilidad, libertad para innovar; aceptación de la diversidad, inclusión, autonomía y, sobre todo, una cultura consolidada de garantías de mejora continua hacia el interior de la organización educativa y de reconocimiento social. Debe tenerse en cuenta que, internacionalmente, se promueven nuevos procesos de acreditación caracterizados por empujar y no por juzgar (Biemiller, 2000), por un aumento de la colaboración entre la institución y la entidad acreditadora y, en resumen, asegurar la calidad mediante la verificación de que una institución se esfuerza, continua y conscientemente, en mejorar los sistemas y procesos de formación de sus estudiantes.

\section{REFERENCIAS}

Asamblea Nacional del Ecuador. Ley Orgánica de Educación Superior. Registro Oficial RO 298, Suplemento del 2010-10-12 (2010)

Becket, N., Brookes, M. Quality management practice in higher education-What quality are we actually enhancing? doi:10.3794/johlste.71.174, Journal of Hospitality, Leisure, Sport \& Tourism Education, (en línea), 7(1), 44-54 (2008)

Biemiller, L. Accrediting group changes standards to allow colleges to set their own goals. Chronicle of Higher Education, 46(33), A43 (2000) https://www.ebsco.com/ (ERIC database). Acceso: 4 de junio 2017

CEAACES. Informe General de la Evaluación de los Institutos Superiores Técnicos y Tecnológicos. https://goo.gl/C2WQPV (en línea). Acceso: 9 de septiembre (2016a)

CEAACES. Reglamento para la determinación de resultados de los ISTT (Institutos Superiores Técnicos y Tecnológicos). https://goo.gl/w3aZbw (en línea). Acceso: 9 septiembre (2016b) 
CEAACES. Informe General de la Evaluación de los Institutos Superiores Técnicos y Tecnológicos. Tomado de: Informe Final de la Evaluación del Entorno de Aprendizaje de los Institutos Superiores Técnicos y Tecnológicos. Instituto Tecnológico Superior Bolivariano de Tecnología (2397) (copia dura confidencial) (2016c)

Diario: El Telégrafo (Ecuador). Artículo de la Redacción Sociedad: " 5 de 8 universidades cofinanciadas usaron el $100 \%$ del presupuesto estatal para becas". https://goo.gl/fFXKNs. Fecha de publicación: 1 de abril (2016), Acceso: 25 de agosto (2016)

Harvey, L. y Williams J. Fifteen Years of Quality in Higher Education. doi:10.1080/13538321003679457. Quality in Higher Education, 16(1), 3-36, April (2010)

Hopkins, D., Mel, A., West, M. School Improvement in an Era of Change. Londres, Cassell (1994)

House, E.R. La evaluación, ética y poder. Madrid, Morata (2004)

Hoyos, G. Participación del estado, de la comunidad académica y de la sociedad en el mejoramiento de la calidad de la educación superior. Derecho y Realidad, 1(22), 135-152 (2016)

Lemaitre, M.J. y Zenteño, M.E. Aseguramiento de la calidad en Iberoamérica. Educación Superior. Informe 2012, CINDA, Santiago de Chile (2012)

Loukkola, T. Examining quality culture: part 1. Quality assurance processes Higher Education Institutions, European University Association, Bruselas (2010)

Morse, J. Accreditation and Quality Assurance from the Perspective of a U.S. Regional Accrediting Agency. Third Biennial Conference of the Chinese Association for Institutional Research, Guangshou, China, July 6-9 (2008), https://goo.gl/pwGjyL. Acceso: 30 de junio (2017)

Natarajan, R. The Role of Accreditation in Promoting Quality Assurance of Technical Education. International Journal of Engineering Education, 16(2), 85-96 (2000)

Ramírez, P.E., Alfaro, J.L., Evaluación de la Eficiencia de las Universidades pertenecientes al Consejo de Rectores de las Universidades Chilenas: Resultados de un Análisis Envolvente de Datos. Doi:10.4067/S0718-50062013000300005, Formación Universitaria, 6(3), 31-38 (2013). Acceso: 3 de septiembre (2016)

Redon, S. Autoevaluación institucional y acreditación como aseguramiento de la calidad de la educación: implicancias teóricas y prácticas. Doi:10.4067/S0718-07052009000200016. Estudios pedagógicos (Valdivia), 35(2), 269-284 (2009)

Stufflebeam D.L. Introduction: Recommendations for improving evaluations in U.S. public schools. Studies in Educational Evaluation, 20(1), 3-21 (1994)

Tyler, L.A. y Bernasconi, A., Evaluation of Higher Education in Latin America: Three Orders of Magnitude. Conference on Educational Reform in Central America, Panama and the Dominican Republic held, El Salvador, enero $25-26$ (1999)

Universidad de las Artes. La educación superior en el Ecuador a cinco años de la LOES. Artículo digital. https://goo.gl/HJK8dv, 14 de Octubre (2015). Acceso: 10 de septiembre (2016)

Villacis, J., El Estado financia a 30 universidades públicas. (Artículo Digital) 5 febrero (2016) https://goo.gl/w1CA1V. Acceso: 3 de septiembre (2016) 